\title{
Cryptographic key distribution and authentication protocols for secure group communication
}

\author{
A.C.M. Hutchison and K. Bauknecht \\ University of Zürich, Department of Computer Science \\ Winterthurerstr. 190, CH-8057 Switzerland, \\ Telephone: +41.1 .25745 11. Fax: +41.1 .3630035$. \\ email \{hutch, baukn\}@ifi.unizh.ch
}

\begin{abstract}
Authentication protocols have until now focussed on two or three party interaction and neglected $n$ party interactions as in the case of more general group communication. In this discourse, the semantics of group authentication are addressed and a separation into complete and selective group authentication techniques is proposed.
\end{abstract}

\section{Keywords}

Authentication, cryptographic key distribution, group security

\section{INTRODUCTION}

The recent emergence of groupware (software supporting group interaction) has raised associated requirements for the provision of group security. One of the primary security problems when considering group interaction is that of authentication. Authentication is an interchange through which parties verify the claimed identities of other parties. While the meaning and mechanics of such an interchange are well understood for two and three party involvement, the semantics of group authentication are less developed.

\section{CRYPTOGRAPHIC KEYS FOR SECURE GROUP COMMUNICATION}

In a two-party interaction it is typically the case that a shared session key is established during an authentication interchange. This key can then be used to secure subsequent exchanges between the parties. In a group environment there are various possibilities for the type of cryptographic key deployed. A single shared group key is one possibility, but pairwise shared keys, or distributed keys (as in a threshold scheme) are other possibilities. 
Different security and performance levels can be achieved within a group according to the cryptographic scheme(s) chosen.

\section{GROUP AUTHENTICATION SEMANTICS}

In two party authentication the primary goal is that each party confirms the identity of the other. In a group there are several possibilities which can be selected from according to what should be achieved by the group authentication. The most stringent form of group authentication which can be identified is where each member of the group proves its identity to each other member of the group. This is the logical extension of two party authentication and we use the term complete group authentication to describe this.

It should be recognised that group membership fluctuates in a dynamic group environment (such as computer conferencing), with parties joining and leaving the group. Upon the arrival of a new member in such a group, one possibility is to conduct another complete group authentication. This would be inefficient though, since existing members have already resolved their mutual suspicion of one another. Here selective group authentication can be conducted and we can distinguish incremental and single selective sub-categories.

Incremental selective authentication proceeds with a joining group member interacting with all existing group members. Existing group members do not interact with each other, however, since they typically conducted a complete group authentication at the time of group establishment.

Single selective group authentication occurs when authentication between a new member and the group occurs via a single gatekeeper. In this manner a joining member interacts only with this gatekeeper, and existing members of the group trust this party to verify the joining member's identity and to control admission. The limitations of this approach are that the joining member does not verify existing group members' identities individually, and that existing members have to trust the gatekeeper.

The selection (or combination) of complete and selective authentication techniques can be used to provide adequate and efficient group authentication and cryptographic key distribution and we have developed a suite of protocols according to this classification.

\section{CONCLUSION}

There are several approaches by which authentication and key distribution can be extended to groups. Before protocols can be described or selected, it is necessary to determine whether complete or selective group authentication should be applied, according to the particular group security context. As groupware becomes more widespread, so the importance of group security measures becomes more relevant and urgent.

\section{BIOGRAPHY}

Andrew Hutchison is a $\mathrm{PhD}$ student in the Department of Computer Science at the University of Zürich. Kurt Bauknecht is a Professor in, and the Director of, the Department of Computer Science at the University of Zürich. 